\title{
Some Properties of the Sorgenfrey Line and the Sorgenfrey Plane
}

\author{
Adam St. Arnaud \\ University of Alberta \\ Edmonton, Canada
}

\author{
Piotr Rudnicki \\ University of Alberta \\ Edmonton, Canada
}

\begin{abstract}
Summary. We first provide a modified version of the proof in 3 that the Sorgenfrey line is $T_{1}$. Here, we prove that it is in fact $T_{2}$, a stronger result. Next, we prove that all subspaces of $\mathbb{R}^{\mathbf{1}}$ (that is the real line with the usual topology) are Lindelöf. We utilize this result in the proof that the Sorgenfrey line is Lindelöf, which is based on the proof found in [8]. Next, we construct the Sorgenfrey plane, as the product topology of the Sorgenfrey line and itself. We prove that the Sorgenfrey plane is not Lindelöf, and therefore the product space of two Lindelöf spaces need not be Lindelöf. Further, we note that the Sorgenfrey line is regular, following from [3]:59. Next, we observe that the Sorgenfrey line is normal since it is both regular and Lindelöf. Finally, we prove that the Sorgenfrey plane is not normal, and hence the product of two normal spaces need not be normal. The proof that the Sorgenfrey plane is not normal and many of the lemmas leading up to this result are modelled after the proof in [3], that the Niemytzki plane is not normal. Information was also gathered from 15.
\end{abstract}

MSC: 54D15 54B10 03B35

Keywords: topological spaces; products of normal spaces; Sorgenfrey line; Sorgenfrey plane; Lindelöf spaces

MML identifier: TOPGEN_6, version: 8.1.01 5.13.1174

The notation and terminology used in this paper have been introduced in the following articles: [16], [1], [13], [12], [11, [14], [19], [18], [9], [2], 10], [3], 17], [20], and [6].

In this paper $T$ denotes a topological space, $x, y, a, b, U, U_{1}, r_{1}$ denote sets, $p, q$ denote rational numbers, $F, G$ denote families of subsets of $T$, and $U_{2}, I$ denote families of subsets of Sorgenfrey line.

Observe that Sorgenfrey line is $T_{2}$.

Now we state the proposition:

(1) Let us consider real numbers $x, a, b$. Suppose $x \in] a, b[$. Then there exist rational numbers $p, r$ such that 
(i) $x \in] p, r[$, and

(ii) $] p, r[\subseteq] a, b[$.

Proof: Consider $p$ being a rational number such that $p>a$ and $x>p$. Consider $r$ being a rational number such that $x<r<b.] p, r[\subseteq] a, b[$.

Let us observe that every subspace of $\mathbb{R}^{\mathbf{1}}$ is Lindelöf and Sorgenfrey line is Lindelöf.

The Sorgenfrey plane yielding a non empty strict topological space is defined by the term

(Def. 1) Sorgenfrey line $\times$ Sorgenfrey line.

The functor real-anti-diagonal yielding a subset of $\mathbb{R} \times \mathbb{R}$ is defined by the term

(Def. 2) $\{\langle x, y\rangle$, where $x, y$ are real numbers : $y=-x\}$.

Now we state the propositions:

(2) $\mathbb{Q} \times \mathbb{Q}$ is a dense subset of the Sorgenfrey plane. Proof: $\mathbb{Q} \times \mathbb{Q} \subseteq \Omega_{\alpha}$, where $\alpha$ is the Sorgenfrey plane by [17, (12)]. Reconsider $C=\mathbb{Q} \times \mathbb{Q}$ as a subset of the Sorgenfrey plane. For every subset $A$ of the Sorgenfrey plane such that $A \neq \emptyset$ and $A$ is open holds $A$ meets $C$ by [16, (5)], [6, (90)], [4, $(31)]$.

(3) $\overline{\overline{\text { real-anti-diagonal }}}=\mathfrak{c}$. Proof: $\mathbb{R} \approx$ real-anti-diagonal by [ 5 , (4)].

(4) real-anti-diagonal is a closed subset of the Sorgenfrey plane. PROof: Set $L=$ real-anti-diagonal. Set $S=$ the Sorgenfrey plane. $L \subseteq \Omega_{S}$. Reconsider $L=$ real-anti-diagonal as a subset of the Sorgenfrey plane. Define $\mathcal{P}$ [element, element] $\equiv$ there exist real numbers $x, y$ such that $\$_{1}=\langle x$, $y$ ) and $\$_{2}=x+y$. For every element $z$ such that $z \in$ the carrier of $S$ there exists an element $u$ such that $u \in$ the carrier of $\mathbb{R}^{\mathbf{1}}$ and $\mathcal{P}[z, u]$ by [7, (17)]. Consider $f$ being a function from $S$ into $\mathbb{R}^{\mathbf{1}}$ such that for every element $z$ such that $z \in$ the carrier of $S$ holds $\mathcal{P}[z, f(z)]$ from [5. Sch. 1]. For every elements $x, y$ of $\mathbb{R}$ such that $\langle x, y\rangle \in$ the carrier of $S$ holds $f(\langle x, y\rangle)=x+y$. For every point $p$ of $S$ and for every positive real number $r$, there exists an open subset $W$ of $S$ such that $p \in W$ and $\left.f^{\circ} W \subseteq\right] f(p)-r, f(p)+r\left[\right.$ by [2, (11)], [16, (6)]. Reconsider $z_{1}=0$ as an element of $\mathbb{R}$. Reconsider $k=\left\{z_{1}\right\}$ as a subset of $\mathbb{R}^{\mathbf{1}} . L=f^{-1}(k)$ by [5, (38)].

(5) Let us consider a subset $A$ of the Sorgenfrey plane.

Suppose $A=$ real-anti-diagonal. Then $\operatorname{Der} A$ is empty.

(6) Every subset of real-anti-diagonal is a closed subset of the Sorgenfrey plane. The theorem is a consequence of (4) and (5).

Note that the Sorgenfrey plane is non Lindelöf and Sorgenfrey line is regular and Sorgenfrey line is normal and the Sorgenfrey plane is non normal. 
Acknowledgement: I would like to thank Piotr Rudnicki for taking me on as his summer student and being a mentor to me. Piotr was an incredibly caring, intelligent, funny, passionate human being. I am proud to know I was his last student, in a long line of students he has mentored and cared about throughout his life. Thank you Piotr, for the opportunity you gave me, and for the faith, confidence and trust you showed in me. I will miss you.

\section{REFERENCES}

[1] Grzegorz Bancerek. Cardinal numbers. Formalized Mathematics, 1(2):377-382, 1990.

[2] Grzegorz Bancerek. On constructing topological spaces and Sorgenfrey line. Formalized Mathematics, 13(1):171-179, 2005.

[3] Grzegorz Bancerek. Niemytzki plane - an example of Tychonoff space which is not $T_{4}$ Formalized Mathematics, 13(4):515-524, 2005.

[4] Grzegorz Bancerek. Bases and refinements of topologies Formalized Mathematics, 7(1): 35-43, 1998.

[5] Czesław Byliński. Functions from a set to a set. Formalized Mathematics, 1(1):153-164, 1990.

[6] Czesław Byliński. Some basic properties of sets. Formalized Mathematics, 1(1):47-53, 1990.

[7] Agata Darmochwał and Yatsuka Nakamura. Metric spaces as topological spaces - fundamental concepts. Formalized Mathematics, 2(4):605-608, 1991.

[8] Ryszard Engelking. Outline of General Topology. North-Holland Publishing Company, 1968.

[9] Adam Grabowski. On the boundary and derivative of a set. Formalized Mathematics, 13 (1):139-146, 2005.

[10] Adam Grabowski. On the Borel families of subsets of topological spaces Formalized Mathematics, 13(4):453-461, 2005.

[11] Andrzej Kondracki. Basic properties of rational numbers Formalized Mathematics, 1(5): 841-845, 1990.

[12] Beata Padlewska and Agata Darmochwał. Topological spaces and continuous functions Formalized Mathematics, 1(1):223-230, 1990.

[13] Karol Pak. Basic properties of metrizable topological spaces. Formalized Mathematics, 17(3):201-205, 2009. doi 10.2478/v10037-009-0024-8

[14] Konrad Raczkowski and Paweł Sadowski. Topological properties of subsets in real numbers. Formalized Mathematics, 1(4):777-780, 1990.

[15] Lynn Arthur Steen and J. Arthur Jr. Seebach. Counterexamples in Topology. SpringerVerlag, 1978.

[16] Andrzej Trybulec. A Borsuk theorem on homotopy types Formalized Mathematics, 2(4): $535-545,1991$.

[17] Andrzej Trybulec. Subsets of complex numbers. Mizar Mathematical Library.

[18] Zinaida Trybulec. Properties of subsets. Formalized Mathematics, 1(1):67-71, 1990.

[19] Edmund Woronowicz. Relations and their basic properties Formalized Mathematics, 1 (1):73-83, 1990.

[20] Mirosław Wysocki and Agata Darmochwał. Subsets of topological spaces Formalized Mathematics, 1(1):231-237, 1990.

Received April 17, 2013 